\title{
CYCLICITY OF SINGULAR INNER FUNCTIONS FROM THE CORONA THEOREM
}

\author{
OMAR EL-FALLAH, KARIM KELLAY, AND KRISTIAN SEIP
}

\begin{abstract}
Carleson's corona theorem is used to obtain two results on cyclicity of singular inner functions in weighted Bergman-type spaces on the unit disk. Our method proof requires no regularity conditions on the weights.
\end{abstract}

\section{INTRODUCTION}

This paper studies cyclicity of singular inner functions in two different classes of weighted Bergman-type spaces. In both cases, our proofs rely crucially on Carleson's corona theorem. An interesting feature of this method of proof is that regularity conditions on the weights can be avoided.

We begin by considering weighted $\ell^{2}$ spaces, viewed as spaces of analytic functions in the unit disk. We say that a sequence of positive numbers $\omega=(\omega(n))_{n \geq 0}$ is a weight sequence if $\omega(n) \nearrow+\infty$ and $\log \omega(n)=o(n)$ when $n \rightarrow \infty$. With every weight sequence $\omega$ we associate the weighted Bergman space $\mathcal{A}_{\omega}^{2}$ which consists of all analytic functions $f(z)=\sum_{n=0}^{\infty} a_{n} z^{n}$ on the open unit disk $\mathbb{D}$ such that

$$
\|f\|_{\omega, 2}^{2}=\sum_{n \geq 0} \frac{\left|a_{n}\right|^{2}}{\omega(n)^{2}}<\infty .
$$

A function $f$ in $\mathcal{A}_{\omega}^{2}$ is said to be cyclic in $\mathcal{A}_{\omega}^{2}$ if the set of functions $p f$ with $p$ a polynomial is dense in $\mathcal{A}_{\omega}^{2}$.

We will prove the following theorem.

Theorem 1. Let $\omega$ be a weight sequence. If

$$
\sum_{n \geq 1} \frac{(\log \omega(n))^{2}}{n^{2}}=\infty
$$

then every function in $H^{\infty}$ without zeros in $\mathbb{D}$ is cyclic in $\mathcal{A}_{\omega}^{2}$;

Date: October 29, 2018.

2000 Mathematics Subject Classification. 30H20; 30H80, 47A16.

Key words and phrases. Weighted Bergman space, cyclic function, singular inner function, corona theorem.

The first author was supported by Egide-Volubilis. The second author was supported by Egide-Volubilis and ANRDynop. The third author was supported by the Research Council of Norway grant 185359/V30. This work started when the third author was visiting CMI, and he thanks LATP for its support and hospitality. 
This result has its roots in work of Beurling [1] and Nikolskii [7, §2.6, Theorem 2]. Requiring a certain regularity condition on $\omega$, Beurling proved that every function in $H^{\infty}$ without zeros in $\mathbb{D}$ is cyclic in $\bigcup_{n \geq 1} \mathcal{A}_{\omega^{n}}^{2}$, equipped with the inductive limit topology, if and only

$$
\sum_{n \geq 1} \frac{\log \omega(n)}{n^{3 / 2}}=\infty .
$$

The Hilbert space case was considered by Nikolskii [7, §2.6, Theorem 2] who proved that whenever $\omega$ is log-concave, i.e., $\left.\omega^{2}(n) \geq \omega(n+1) \omega(n-1)\right)$, the divergence condition (2) implies that every function in $H^{\infty}$ without zeros in $\mathbb{D}$ is cyclic in $\mathcal{A}_{\omega}^{2}$. Beurling used Bernstein's approximation theorem, while Nikolskii relied on a theorem on quasi-analyticity that requires the log-concavity condition. Beurling pointed out at the end of his paper that he could not dispense with a certain convexity condition and that it remained an open problem to obtain a general sufficient condition for cyclicity.

Thus the novelty of Theorem 1, besides the method of proof, is the absence of any regularity condition on $\omega$. In the second remark after Theorem 2 below, we will give an example showing that Theorem 1 enables us to deal with weights that are not covered by Nikolskii's theorem, in spite of the fact that the divergence condition (11) implies (2).

We now turn to our second result, which deals with a situation in which growth restrictions are nonradial. Let $E$ be a closed subset of $\mathbb{T}$ and let $\Lambda$ be a nonincreasing and positive function on $] 0,2]$ such that $\Lambda\left(0^{+}\right)=+\infty$. We denote by $\mathcal{B}_{\Lambda, E}^{\infty}$ the space of all analytic functions $f$ on $\mathbb{D}$ such that

$$
\|f\|_{\Lambda, E, \infty}=\sup _{z \in \mathbb{D}}|f(z)| e^{-\Lambda(\mathrm{d}(z, E))}
$$

here $\mathrm{d}(\cdot, \cdot)$ stands for Euclidean distance on $\mathbb{C}$. Let $I$ be the singular inner function defined by

$$
I(z)=e^{-\frac{1+z}{1-z}}
$$

Gevorkyan and Shamoyan showed in [5] that if $E=\{1\}$ and $\Lambda$ satisfies certain regularity conditions, then $I$ is cyclic in $\mathcal{B}_{\Lambda,\{1\}}^{\infty}$ if and only if $\Lambda$ fails to be integrable. We will now prove the same result without any additional assumption on $\Lambda$.

Theorem 2. The singular inner function I is cyclic in $\mathcal{B}_{\Lambda,\{1\}}^{\infty}$ if and only if

$$
\int_{0}^{2} \Lambda(t) d t=\infty .
$$

Several remarks are in order before we turn to the proofs of our theorems:

1. Cyclicity of the singular inner function $I$ was first considered in weighted approximation theory by Keldyš [4] and Beurling [1]. See also [7, §2.8, Theorem 1]. The idea of using the corona theorem in this context goes back to Roberts [8]. 
2. We next give an example of a weight that satisfies (11) but to which Nikolskii's theorem does not apply. Set

$$
\log \omega(n)=2^{2^{j-1}}, \quad n \in\left[2^{2^{j}}, 2^{2^{j+1}}\right)
$$

for $j=1,2, \ldots$. Then clearly (1) holds, but we may check that if $\widetilde{\omega}$ is log-concave and $\widetilde{\omega} \leq \omega$, then

$$
\sum_{n \geq 1} \frac{\log \widetilde{\omega}(n)}{n^{3 / 2}}<+\infty
$$

To see this, let $g$ be the linear function that satisfies $g\left(2^{2^{j-1}}\right)=2^{2^{j-3}}$ and $g\left(2^{2^{j}}\right)=2^{2^{j-2}}$. By concavity, $\widetilde{\omega}(n) \leq \min \left(2^{2^{j-1}}, g(n)\right)$ for $n$ in the interval $\left[2^{2^{j}}, 2^{2^{j+1}}\right)$. A straightforward computation shows that the piecewise linear function

$$
h(n)=\min \left(2^{2^{j-1}}, g(n)\right), \quad n \in\left[2^{2^{j}}, 2^{2^{j+1}}\right)
$$

satisfies

$$
\sum_{n>4} \frac{h(n)}{n^{3 / 2}}<\infty
$$

3. The assumption that $\omega$ is nondecreasing implies that the shift operator is a contraction on $\mathcal{A}_{\omega}^{2}$, a fact that plays an essential role in the proof of Theorem 1 given below. One may ask if this monotonicity can be dispensed with. While we can not rule out the possibility that it can be relaxed, the following example shows that it can not simply be removed. Namely, let $\omega$ be any sequence such that $\omega(2 n)=1$. Then $U\left(z^{2}\right)$ is not cyclic in $\mathcal{A}_{\omega}^{2}$ when $U$ is an arbitrary inner function. Indeed, if there is a sequence of polynomials $p_{n}$ such that $\left\|p_{n}(z) U\left(z^{2}\right)-1\right\|_{\omega, 2} \rightarrow 0$, we may write $p_{n}(z)=q_{n}\left(z^{2}\right)+z h_{n}\left(z^{2}\right)$; then $\left\|q_{n}\left(z^{2}\right) U\left(z^{2}\right)-1\right\|_{\omega, 2} \rightarrow 0$ which means that $U$ is cyclic in $H^{2}$. But this contradicts Beurling's theorem.

4. We have the following relation between the two kinds of Bergman spaces considered in the present work. We denote by $\mu$ normalized Lebesgue measure on $\mathbb{D}$ and define $\mathcal{B}_{\Lambda, \mathbb{T}}^{2}$ to be the space of all analytic functions $f$ on $\mathbb{D}$ such that

$$
\|f\|_{\Lambda, \mathbb{T}, 2}^{2}=\int_{\mathbb{D}}|f(z)|^{2} e^{-2 \Lambda(1-|z|)} d \mu(z)<\infty .
$$

A simple computation shows that $f(z)=\sum_{n=0}^{\infty} a_{n} z^{n}$ belongs to $\mathcal{B}_{\Lambda, \mathbb{T}}^{2}$ if and only if

$$
\|f\|_{\Lambda, \mathbb{T}, 2}^{2}=\sum_{n \geq 0} \frac{\left|a_{n}\right|^{2}}{\omega(n)^{2}}<\infty
$$

where

$$
\omega(n)^{-2}=\int_{0}^{1} r^{2 n+1} e^{-2 \Lambda(1-|z|)} d r, \quad n \geq 0
$$


Note that this moment sequence $\omega$ is log-concave. Conversely, if $\omega$ is a log-concave weight sequence, then there exists a $\Lambda$ such that

$$
c \omega(n) \leq\left(\int_{0}^{1} r^{2 n+1} e^{-2 \Lambda(1-|z|)} d r\right)^{-1 / 2} \leq C \omega(n)
$$

for positive constants $c$ and $C$ independent of $n \geq 1$ [2, Proposition 4.1]. However, in general, we can not write $\mathcal{A}_{\omega}^{2}$ as $\mathcal{B}_{\Lambda, \mathbb{T}}^{2}$.

5. In [7, $§ 2.6, \S 2.7]$, Nikolskii proved, under some regularity conditions on $\Lambda$, that $I$ is cyclic in $\mathcal{B}_{\Lambda, \mathbb{T}}^{2}$ if and only if

$$
\int_{0}^{2} \sqrt{\frac{\Lambda(t)}{t}} d t=\infty
$$

It is interesting to note when $\Lambda$ and $\omega$ are related as in (4), then, under suitable regularity conditions on $\Lambda$, (15) is equivalent to (2) and (3) is equivalent to (11) [7, §2.6 Lemma 1, Lemma 2]. As will be pointed out in Section 3 below, a slight variant of our proof of Theorem 2 gives that (3) is in fact sufficient for every singular inner function $U$ to be cyclic in $\mathcal{B}_{\Lambda, \mathbb{T}}^{2}$. Note that, again, no additional regularity condition on $\Lambda$ is required.

\section{Proof of Theorem 1}

Our proof of Theorem 1 will rely on three lemmas.

The first lemma gives a convenient reformulation of condition (1) of Theorem 11. We will use only one of the implications of the lemma, but we find the result to be of some general interest and give therefore the simple proof of the full equivalence between the two conditions.

Lemma 1. Let $\omega$ be a weight sequence. Then the divergence condition (11) of Theorem 1 holds if and only if there exists a sequence of nonnegative integers $\left(n_{j}\right)_{j \geq 0}$ such that $\log \omega\left(n_{j+1}\right) \geq 2 \log \omega\left(n_{j}\right)$ and

$$
\sum_{j \geq 0} \frac{\left(\log \omega\left(n_{j}\right)\right)^{2}}{n_{j}}=\infty
$$

Proof. If (11) holds, then we define $n_{j}$ inductively by setting $n_{0}=1$ and requiring

$$
n_{j+1}=\min \left\{n>n_{j}: \log \omega(n) \geq 2 \log \omega\left(n_{j}\right)\right\} .
$$

Since

$$
\sum_{n=n_{j}}^{n_{j+1}-1} \frac{(\log \omega(n))^{2}}{n^{2}} \leq 4\left(\log \omega\left(n_{j}\right)\right)^{2} \sum_{n=n_{j}}^{n_{j+1}-1} \frac{1}{n^{2}} \leq 4 \frac{\left(\log \omega\left(n_{j}\right)\right)^{2}}{n_{j}-1}
$$

we conclude that condition (1) of Theorem 1 implies (6).

To prove the reverse implication, we observe that if $\log \omega\left(n_{j+1}\right) \geq 2 \log \omega\left(n_{j}\right)$ for every $j$, then

$$
\sum_{l: \frac{n_{j}}{2} \leq n_{l} \leq n_{j}} \frac{\left(\log \omega\left(n_{l}\right)\right)^{2}}{n_{l}} \leq 4 \frac{\left(\log \omega\left(n_{j}\right)\right)^{2}}{n_{j}}
$$


Thus we may assume that the sequence $\left(n_{j}\right)$ satisfies the exponential growth condition $n_{j+1} \geq 2 n_{j}$, in which case we have

$$
\frac{\left(\log \omega\left(n_{j}\right)\right)^{2}}{n_{j}} \leq 4 \sum_{n=n_{j}}^{n_{j+1}-1} \frac{(\log \omega(n))^{2}}{n^{2}}
$$

and so (6) implies the divergence condition (11) of Theorem 1 .

Since $\omega$ is nondecreasing, the shift operator $S$ defined by $S f(z)=z f(z)$ is a contraction on $\mathcal{A}_{\omega}^{2}$. It follows that for $f$ in $H^{\infty}, f(S)$ makes sense by $H^{\infty}$ functional calculus. Therefore, by von Neumann's inequality, we have

$$
\|f \varphi\|_{\omega, 2}=\|f(S) \varphi\|_{\omega, 2} \leq\|f(S)\|\|\varphi\|_{\omega, 2} \leq\|f\|_{\infty}\|\varphi\|_{\omega, 2}
$$

for every $\varphi$ in $\mathcal{A}_{\omega}^{2}$. The next lemma is a simple consequence of this fact.

Lemma 2. Let $U$ be a singular inner function and $\lambda$ a positive number. Then $U$ is cyclic in $\mathcal{A}_{\omega}^{2}$ if and only if $U^{\lambda}$ is cyclic in $\mathcal{A}_{\omega}^{2}$.

Proof. Since functions in $H^{\infty}$ are multipliers, it suffices to prove the lemma in the special case when $\lambda=2$.

Suppose first that $U$ is cyclic. Then there exist polynomials $p_{n}$ such that $\left\|1-p_{n} U\right\|_{\omega, 2} \rightarrow 0$ as $n \rightarrow \infty$. So $\left\|U-p_{n} U^{2}\right\|_{\omega, 2} \leq\|U\|_{\infty}\left\|1-p_{n} U\right\|_{\omega, 2} \rightarrow 0$ and $U^{2}$ is cyclic.

We now assume that $U^{2}$ is cyclic. Then there exist polynomials $p_{n}$ such that $\| 1-$ $p_{n} U^{2} \|_{\omega, 2} \rightarrow 0$ when $n \rightarrow \infty$. So the functions $f_{n}=p_{n} U$ in $H^{\infty}$ have the property that $\left\|1-f_{n} U\right\|_{\omega, 2} \rightarrow 0$, which means that $U$ is cyclic since $\omega(n) \rightarrow \infty$, we have

$$
\overline{\{p V: p \text { polynomial }}^{\mathcal{A}_{\omega}^{2}}={\overline{\left\{f V: f \in H^{\infty}\right\}}}_{\mathcal{A}_{\omega}^{2}}
$$

for every function $V$ in $H^{\infty}$.

We now turn to our application of the corona theorem.

Lemma 3. Let $\nu$ be the singular measure of $U$ and set $c^{2}=\nu(\mathbb{T})$. Then for every nonnegative integer $n$, there is a function $f_{n}$ in $H^{\infty}$ such that

$$
\begin{aligned}
\left\|1-f_{n} U\right\|_{\omega, 2} & \leq \frac{e^{A(c \sqrt{n}+1)}}{\omega(n)}, \\
\left\|f_{n}\right\|_{\infty} & \leq e^{A(c \sqrt{n}+1)}
\end{aligned}
$$

with $A$ an absolute constant.

Proof. Note that

$$
\begin{aligned}
\inf _{z \in \mathbb{D}}\left[|U(z)|+|z|^{n}\right] & \geq \inf _{z \in \mathbb{D}}\left[\exp \frac{-2 c^{2}}{1-|z|}+|z|^{n}\right] \\
& \geq e^{-2 c \sqrt{n}}=\delta_{n} .
\end{aligned}
$$


By Carleson's corona theorem [3], [6, p. 66], there exist $f_{n}, g_{n} \in H^{\infty}$ such that

$$
\left\{\begin{array}{l}
f_{n} U+z^{n} g_{n}=1 \\
\left\|f_{n}\right\|_{\infty} \leq 2^{5} \delta_{n}^{-3}, \quad\left\|g_{n}\right\|_{\infty} \leq 2^{5} \delta_{n}^{-3}
\end{array}\right.
$$

which implies that (8) is satisfied for some absolute positive constant $A$. We observe that (77) also holds by the observation above and the estimate

$$
\left\|1-f_{n} U\right\|_{\omega, 2}=\left\|z^{n} g_{n}\right\|_{\omega, 2} \leq\left\|g_{n}\right\|_{\infty}\left\|z^{n}\right\|_{\omega, 2}
$$

Proof of Theorem 1. Let $f$ be a function in $H^{\infty}$ without any zeros in $\mathbb{D}$. We write $f=F U$, where $F$ is an outer function and $U$ is a singular inner function with associated singular measure $\nu$. Since $F$ is outer, $F$ is cyclic in $H^{2} \subset \mathcal{A}_{\omega}^{2}$. Hence it remains only to prove that $U$ is cyclic in $\mathcal{A}_{\omega}^{2}$.

Let $m_{1}, m_{2}, \ldots, m_{N}$ be arbitrary positive integers and $\lambda_{j}$ associated positive numbers such that

$$
\sum_{j=1}^{N} \lambda_{j}^{2}=1
$$

Set $U_{j}=U^{\lambda_{j}^{2}}$ and let $f_{j}$ be a function such that

$$
\left\{\begin{array}{l}
\left\|1-f_{j} U_{j}\right\|_{\omega, 2} \leq \exp \left[A\left(c \lambda_{j} \sqrt{m_{j}}+1\right)-\log \omega\left(m_{j}\right)\right] \\
\left\|f_{j}\right\|_{\infty} \leq e^{A\left(c \lambda_{j} \sqrt{m_{j}}+1\right)}, \quad j=1, \ldots, N
\end{array}\right.
$$

By Lemma 3, such a function exists for every $j$, with $A$ an absolute constant. Since

$$
1-U \prod_{j=1}^{N} f_{j}=1-U_{1} f_{1}+U_{1} f_{1}\left(1-U_{2} f_{2}\right)+\ldots+\prod_{j=1}^{N-1} U_{j} f_{j}\left(1-U_{N} f_{N}\right),
$$

we get

$$
\left\|1-U \prod_{j=1}^{N} f_{j}\right\|_{\omega, 2} \leq \sum_{j=1}^{N} \exp \left[\sum_{k=1}^{j} A\left(c \lambda_{k} \sqrt{m_{k}}+1\right)-\log \omega\left(m_{j}\right)\right] .
$$

Now choose $m_{j}=n_{j+j_{0}}$ for some $j_{0}$, where $\left(n_{j}\right)$ is the sequence obtained from Lemma 1 . This means that $\log \omega\left(n_{j+1}\right) \geq 2 \log \omega\left(n_{j}\right)$ and also that (6) holds. Let $N=N\left(j_{0}\right)$ be such that

$$
N=\min \left\{M: \sum_{j=1}^{M} \frac{\left(\log \omega\left(n_{j+j_{0}}\right)\right)^{2}}{n_{j+j_{0}}} \geq(4 A c)^{2}\right\}
$$

and set

$$
\lambda_{j}=\frac{\log \omega\left(n_{j_{0}+j}\right)}{\sqrt{n_{j_{0}+j}}}\left(\sum_{k=1}^{N} \frac{\left(\log \omega\left(n_{j_{0}+k}\right)\right)^{2}}{n_{j_{0}+k}}\right)^{-1 / 2} .
$$


By our choice of sequence $\left(n_{j}\right)$, we have then

$$
\lambda_{j} \leq \frac{1}{4 A c} \frac{\log \omega\left(n_{j_{0}+j}\right)}{\sqrt{n_{j_{0}+j}}} \text { and } \sum_{k=1}^{j} \log \omega\left(n_{j_{0}+k}\right) \leq 2 \log \omega\left(n_{j_{0}+j}\right) .
$$

Thus we get

$$
\left\|1-U \prod_{j=1}^{N} f_{j}\right\|_{\omega, 2} \leq \sum_{j=1}^{N} e^{A j-\frac{1}{2} \log \omega\left(n_{j_{0}+j}\right)} \leq \frac{C}{\sqrt{\omega\left(n_{j_{0}+1}\right)}}
$$

for an absolute constant $C$. This finishes the proof since $\omega\left(n_{j_{0}+1}\right) \rightarrow \infty$ when $j_{0} \rightarrow \infty$.

\section{Proof of Theorem 2}

For the proof of Theorem 2, we need the following two lemmas.

Lemma 4. Suppose that $0<\delta<1$ and let $f_{\delta}$ be the outer function defined by

$$
f_{\delta}(z)=\exp \left(-\Lambda(\delta) \int_{\delta<|t|<\pi} \frac{e^{i t}+z}{e^{i t}-z} d t\right) .
$$

If $a$ is in $\left.] 0,(2 \pi)^{-1}\right]$, then we have

$$
\begin{aligned}
\left|f_{\delta}(z)\right| & \geq e^{-4 \pi^{2} \Lambda(\delta) a} \text { for } \frac{|1-z|^{2}}{1-|z|^{2}} \leq a \delta, \\
\left\|f_{\delta}\right\|_{\Lambda,\{1\}, \infty} & \leq e^{-\pi \Lambda(\delta)} .
\end{aligned}
$$

Proof. We first prove (9)). Let $z$ be a point in $\mathbb{D}$ such that $\frac{|1-z|^{2}}{1-|z|^{2}} \leq a \delta$. Then we have $1-|z| \leq|1-z| \leq 2 a \delta$, which implies that

$$
\log \left|f_{\delta}(z)\right|^{-1}=\Lambda(\delta) \int_{\delta<|t|<\pi} \frac{1-|z|^{2}}{\left|e^{i t}-z\right|^{2}} d t \leq 8 a \delta \Lambda(\delta) \int_{\delta<t<\pi} \frac{d t}{\left(\left|e^{i t}-1\right|-2 a \delta\right)^{2}}
$$

Using that $\left|e^{i t}-1\right| \geq 2 t / \pi$ for $0 \leq t \leq \pi$ and that $a \leq(2 \pi)^{-1}$, we obtain the desired estimate (9).

We will now prove (10); we will do this by showing that for every $z$ in $\mathbb{D}$, we have

$$
\left|f_{\delta}(z)\right| \leq e^{-\pi \Lambda(\delta)+\Lambda(|1-z|)} .
$$

When $|1-z| \leq \delta$, this inequality holds because $\left|f_{\delta}(z)\right| \leq 1$ and $\Lambda$ is a decreasing function. To deal with the case $|1-z|>\delta$, we argue as follows. Let $E_{\delta}$ be the sub-arc of points $e^{i t}$ on the unit circle satisfying $\delta \leq|t| \leq \pi$. Then we may write

$$
\log \left|f_{\delta}(z)\right|=-\Lambda(\delta) \int_{\delta<|t|<\pi} \frac{1-|z|^{2}}{\left|e^{i t}-z\right|^{2}} d t=-\Lambda(\delta) 2 \pi \varpi\left(z, E_{\delta}, \mathbb{D}\right),
$$

where $\varpi\left(z, E_{\delta}, \mathbb{D}\right)$ denotes harmonic measure of $E_{\delta}$ at $z$ in $\mathbb{D}$. A simple geometric argument shows that when $|z-1|>\delta$, z lies in the domain bounded by $E_{\delta}$ and the hyperbolic geodesic between the endpoints of $E_{\delta}$. Therefore, $\varpi\left(z, E_{\delta}, \mathbb{D}\right) \geq 1 / 2$, and (11) follows. 
Lemma 5. Let $c$ be a positive number and $n$ a positive integer such that $4 \pi^{2} c n \leq \Lambda(1 / n)$, and set $I_{c}(z)=e^{-c \frac{1+z}{1-z}}$. Then there exists a bounded analytic function $g_{n}$ such that

$$
\begin{aligned}
\left\|1-g_{n} I_{c}\right\|_{\Lambda,\{1\}, \infty} & \leq e^{B \sqrt{c n \Lambda(1 / n)}-\pi \Lambda(1 / n)}, \\
\left\|g_{n}\right\|_{\infty} & \leq e^{B \sqrt{c n \Lambda(1 / n)}}
\end{aligned}
$$

where $B$ is a universal constant.

Proof. Applying Lemma 4 with $\delta=\delta_{n}=1 / n$ and $a=\sqrt{\frac{c n}{\Lambda(1 / n)}}$, we obtain

$$
\left|f_{\delta_{n}}(z)\right|+\left|I_{c}(z)\right| \geq \min \left(e^{-4 \pi^{2} a \Lambda(\delta)}, e^{-\frac{c}{a \delta}}\right)=e^{-4 \pi^{2} \sqrt{c n \Lambda(1 / n)}}
$$

and

$$
\left\|f_{\delta_{n}}\right\|_{\Lambda,\{1\}, \infty} \leq e^{-\pi \Lambda(1 / n)}
$$

By the corona theorem, we obtain the desired estimates.

Proof of Theorem 2. Assume first that $\Lambda$ is integrable. We will use Keldyš's method [4, 7] to prove that this implies that $I$ is not cyclic. So suppose to the contrary that $I$ is cyclic. Then there exists a sequence of polynomials $\left(p_{n}\right)$ such that $\left\|I p_{n}-1\right\|_{\Lambda,\{1\}, \infty} \rightarrow 0$. Thus, in particular, we have $C=\sup _{n}\left\|I p_{n}\right\|_{\Lambda,\{1\}, \infty}<\infty$. Since $\left|I^{*}(\zeta)\right|=1$ for all $\zeta$ in $\mathbb{T} \backslash\{1\}$, we obtain $\left|p_{n}(\zeta)\right| \leq C e^{-\Lambda(|1-\zeta|)}$. Let $F$ be the outer function given by

$$
F(z)=\exp \left(-\int_{0}^{2 \pi} \frac{e^{i t}+z}{e^{i t}-z} \Lambda\left(\left|1-e^{i t}\right|\right) \frac{d t}{2 \pi}\right) .
$$

By the generalized maximum principal, $\left|p_{n}(z)\right| \leq C|F(z)|$ for all $|z|<1$. But we also have $\lim _{n \rightarrow \infty} p_{n}(z)=1 /|I(z)|$, so that $|I(z)|^{-1} \leq|F(z)|$, but this is impossible since $I$ is a singular inner function and $F$ is an outer function.

The proof of the converse is essentially the same as the proof of Theorem 1, and we will therefore only sketch the argument. We begin by noting that

$$
\int_{0}^{2} \Lambda(t) d t=\infty \quad \Leftrightarrow \quad \sum_{n \geq 1} \frac{\Lambda(1 / n)}{n^{2}}=\infty .
$$

So, by Lemma 1, there exists a sequence $\left(n_{j}\right)$ such that

$$
\Lambda\left(n_{j+1}\right) \geq 2 \Lambda\left(n_{j}\right) \text { and } \sum_{n \geq 1} \frac{\Lambda\left(1 / n_{j}\right)}{n_{j}}=\infty .
$$

We fix $j_{0}$ and choose $N$ so large that

$$
\sum_{j=j_{0}+1}^{N} \frac{\Lambda\left(1 / n_{j}\right)}{n_{j}} \geq 4 \pi^{2} B^{2}
$$

Following the scheme of proof for Theorem 1, we make the choice

$$
U_{j}=I_{\lambda_{j}^{2}}
$$


where

$$
\lambda_{j}^{2}=\frac{\Lambda\left(1 / n_{j}\right)}{n_{j}}\left(\sum_{k=1}^{N} \frac{\Lambda\left(1 / n_{j_{0}+k}\right)}{n_{j_{0}+k}}\right)^{-1} .
$$

By our assumption (12), Lemma $\left[5\right.$ applies with $c=\lambda_{j}^{2}$ and $n=n_{j}$ for $j_{0}+1 \leq j \leq j_{0}+N$. The rest of the proof follows step by step the last part of the proof of Theorem 1 . We omit the details.

Let us note that if we in the latter argument replace the function $f_{\delta_{n}}$ in Lemma 5 by $z^{[n \Lambda(1 / n)]}$ (here $[x]$ denotes the integer part of $x$ ), then we obtain the result mentioned in Remark 5 of the introduction: If $\Lambda$ fails to be integrable, then every singular inner function is cyclic in $\mathcal{B}_{\Lambda, \mathbb{T}}^{\infty}$.

We finally mention that, by the same method of proof, we may replace $\mathcal{B}_{\Lambda,\{1\}}^{\infty}$ in Theorem 2 by $\mathcal{B}_{\Lambda,\{1\}}^{2}$, which is the Hilbert space of analytic functions $f$ on $\mathbb{D}$ such that

$$
\|f\|_{\Lambda,\{1\}, 2}^{2}=\int_{\mathbb{D}}|f(z)|^{2} e^{-2 \Lambda(|1-z|)} d \mu(z)<\infty .
$$

\section{REFERENCES}

[1] A. Beurling, A critical topology in harmonic analysis on semigroups, Acta Math. 112 (1964) $215-228$.

[2] A. Borichev; H. Hedenmalm, Completeness of translates in weighted spaces on the half-line, Acta Math. 174 (1995), 1-84.

[3] L. Carleson, Interpolations by bounded analytic functions and the corona problem, Ann. of Math. 76 (1962), 547-559.

[4] M. V. Keldyš, Sur l'approximation en moyenne par polynomes des fonctions d'une variable complexe, Mat. Sb. 16 (58) (1945), 1-20.

[5] I.M. Gevorkyan; F.A. Shamoyan, Weak invertibility in spaces of functions, analytic in the disk, which permit growth near its boundary, Akad. Nauk Armyan. SSR Dokl. 82 (1986), 156-159.

[6] N. K. Nikolskii, Treatise on the Shift Operator, Springer-Verlag, Berlin, 1986.

[7] N. K. Nikolskii, Selected Problems of Weighted Approximation and Analysis, Proc. Steklov. Inst. Math. 120 (1974); Amer. Math. Soc., Providence, R.I., 1976.

[8] J. W. Roberts, Cyclic inner functions in the Bergman spaces and weak outer functions in $H^{p}$, $0<$ $p<1$, Illinois J. Math. 29 (1985), 25-38.

Département de Mathématiques, Université Mohamed V, B. P. 1014 Rabat, Morocco

E-mail address: elfallah@fsr.ac.ma

CMi, LAtP, Université de Provence, 39, rue F. Joliot-Curie, 13453 Marseille Cedex 13, FRANCE

E-mail address: kellay@cmi.univ-mrs.fr

Department of Mathematical Sciences, Norwegian University of Science and TechnolOGY, NO-7491 Trondheim, NorWAY

E-mail address: seip@math.ntnu.no 